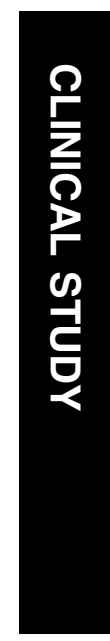

\title{
Can we predict which patients are at risk of having an ungradeable digital image for screening for diabetic retinopathy?
}

\begin{abstract}
Purpose We aimed to determine the reasons for, and variables which predicted, ungradeable retinal photographs during screening patients for diabetic retinopathy. Materials and methods Age, duration of diabetes, visual acuity, and HbA1c were recorded. Following dark adaptation, a single $45^{\circ}$ nonmydriatic photograph was taken of each fundus. The pupils were then dilated and the photograph repeated. Using slit lamp biomicroscopy, lenticular changes (LOCS III), and fundus appearance were recorded.

Results In ungradeable photographs the fovea could not be visualised in $98 \%$ of cases of images from nonmydriatic photography, and in $88 \%$ if mydriasis was used. Poor definition in the nonmydriatic image was associated with a subsequent ungradeable mydriatic photograph $(P=0.001)$, however, the positive predictive value was poor (34\%). Age, posterior subcapsular cataract, and near vision predicted ungradeable status of nonmydriatic photographs $(P<\mathbf{0 . 0 0 1}$, $P=0.004, P=0.006$, respectively; regression analysis). Nuclear colour and poor definition of the nonmydriatic photograph predicted ungradeable status of mydriatic photographs ( $P=0.006 \& P=0.001$, respectively).

Conclusion Inability to visualise the fovea is the commonest cause of an ungradeable image from digital retinal photography. Age and posterior subcapsular cataract were best predictors of ungradeable status of nonmydriatic fundus photographs. Nuclear colour was the strongest predictor for ungradeable mydriatic photography.
\end{abstract}

Eye (2008) 22, 344-348; doi:10.1038/sj.eye.6702611; published online 6 October 2006
H Murgatroyd ${ }^{1}$, A Cox ${ }^{1}$, A Ellingford ${ }^{2}$, JD Ellis ${ }^{1}$, CJ MacEwen ${ }^{1}$ and GP Leese ${ }^{2}$
Keywords: diabetic retinopathy; screening; digital photograph

\section{Introduction}

Diabetic retinopathy is the single largest cause of preventable blindness in the working population in the Western world. ${ }^{1,2}$ Screening for diabetic retinopathy has been shown to reduce visual loss and be cost efficient. ${ }^{3,4}$ Camera-based systems for screening for diabetic retinopathy have been proposed in the UK and North America. ${ }^{5,6}$ In Scotland, the Health Technology Board (HTBS), a body which looks at clinical and cost effectiveness, patient and organisational issues, has proposed a nonmydriatic single digital photograph for screening. ${ }^{7}$ If an unsatisfactory nonmydriatic photograph is obtained then the patient, undergoes immediate pupillary dilatation with $1 \%$ tropicamide and the photograph repeated: 'staged mydriasis'. Using this protocol $75-80 \%$ do not require mydriasis. 8,9 The routine use of mydriasis in digital retinal photography does improve the rate of gradable photographs. Mydriasis, however, increases screening time and can cause inconvenience to the patient especially those needing to drive and/or return to work. ${ }^{10-12}$ We have assessed the detailed reasons, and predictors for an ungradeable nonmydriatic image. Predicting patients who fail nonmydriatic photography could help make screening more efficient by anticipating the need for mydriasis.

Materials and methods

Subjects

Ethical approval was obtained from the Tayside Regional Ethics Committee. Patients were 
recruited consecutively from those attending the medical and ophthalmic diabetes clinics. Patients were excluded from the study if they were unable to give informed consent, were unable to position at the slit lamp table, or were unable to fixate on the light target of the camera.

\section{Protocol}

For all patients age, duration of diabetes, and corrected Snellen visual acuity for near and distance were recorded. Following dark adaptation, a trained photographer (AE) took a single nonmydriatic $45^{\circ}$ field retinal photograph centred on the fovea of each fundus. On each occasion the right eye was photographed before the left and up to $10 \mathrm{~min}$ was allowed between each photograph to allow redilation. Two drops of tropicamide $1 \%$ were then instilled into each eye to produce pharmacological mydriasis.

After $20 \mathrm{~min}$, the patients were examined with a slit lamp biomicroscope by a single trained ophthalmologist (HM). The fundal features were recorded using the former NSC recommendations (described in previous paper by Murgatroyd et $a l^{9}$, personal communication). At the time of the study protocol being set up the recommendations were deemed as the most appropriate. Lenticular changes were recorded using the LOCS III scale. ${ }^{13}$ The LOCS III cataract grading system uses slit lamp photographs to grade nuclear colour, nuclear opalescence, and presence of cortical cataract and/or posterior subcapsular cataract. Cataract severity is graded on a decimal scale and the standards have regularly spaced intervals. Finally, a second fundal photograph was taken through the dilated pupils.

The digitally stored images were presented at full capture resolution randomly to one of two retinal readers, one Ophthalmologist (CM) and one Diabetologist (GL). The retinal photographs were stored as bitmap images and viewed in a darkened room, on CRT screens with a resolution of $1024 \times 768$ pixels at $100 \%$ scale. The retinal readers were masked to any clinical information and whether mydriasis had been used. Three qualities were used to define gradable status (Table 1), and each was assessed independently.

\section{Statistical analysis}

Data were analysed using an SPSS database. Binary logistic regression was used to determine which factors were predictive of ungradeable nonmydriatic and mydriatic photography images. In order to deal with multicolinearity a stepwise entry method was used (likelihood ratio).
Table 1 Defines the criteria to assess image quality

\begin{tabular}{ll}
\hline Criteria judged & $\begin{array}{l}\text { Acceptance } \\
\text { for grading }\end{array}$ \\
\hline $\begin{array}{l}\text { Definition } \\
\text { Good }\end{array}$ & \\
$\quad$ All features fully assessed & Accept \\
Moderate & \\
$\quad$ Some haziness of some small vessels & Accept \\
Poor & Reject \\
Unable to define small vessels & \\
& \\
Field of image & Accept \\
Full image seen & Accept \\
$>3 / 4$ image seen & Reject \\
$<3 / 4$, but $>1 / 2$ image seen & Reject \\
$<1 / 2$ of image seen & \\
Fovea seen & \\
Yes & Accept \\
No & Reject \\
\hline
\end{tabular}

Photographs were deemed ungradeable if any of the criteria above resulted in rejection.

\section{Results}

Informed consent was secured from 398 patients (794 eyes) of 407 patients approached. The median age of patients enroled was 63.0 years (range 17-88, interquartile range 51.8-70.3) with 57\% male. Median duration of diabetes was 7 years (range 1-47, interquartile range 3-13 years), and 35\% were treated with insulin.

Figure 1 illustrates the criteria identified to define each ungradeable nonmydriatic photograph and mydriatic photograph, respectively. Complete data for criteria for ungradeable status was available for $85 \%$ (178/210 eyes) of the ungradeable nonmydriatic photographs and $83 \%$ (33/40 eyes) for the ungradeable mydriatic photographs. In ungradeable images, taken by nonmydriatic photography, the fovea could not be seen in $98 \%$, less than $3 / 4$ of the field could be seen in $54 \%$, and the definition was inadequate for grading in $26 \%$. The corresponding figures for ungradeable images after mydriasis were 88,39 , and $73 \%$ (Figure 1). Characteristics of patients with missing data were similar to those with complete data, except a greater proportion of the latter were taking insulin (Table 2). This suggests there was no important bias between the two groups. The proportion of referrable pathology in ungradeable and gradable photographs for nonmydriatic $(23 \%$ vs $21 \% ; P=0.54)$ and mydriatic photographs (17 vs $21 \%$; $P=0.56$ ) were also not different.

The fovea not being visualised or less than $3 / 4$ of the field being seen after nonmydriatic photography was not associated with a similar finding after mydriatic photography. A nonmydriatic photograph being 
a

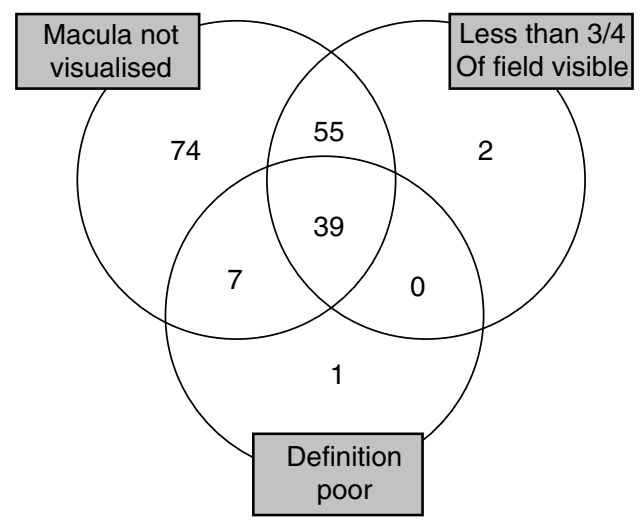

b

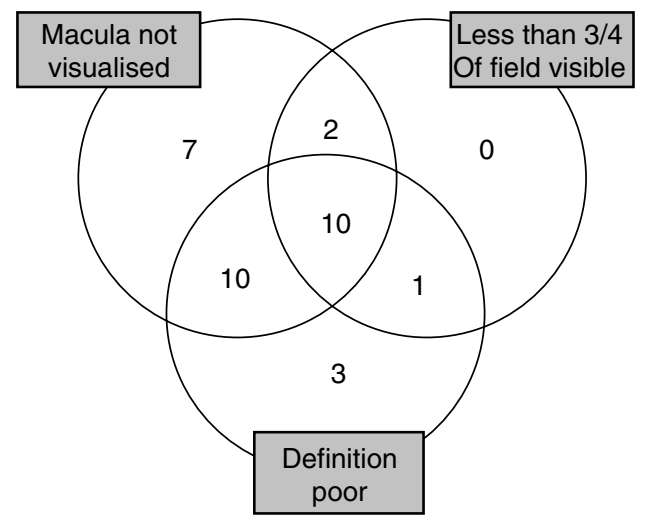

Figure 1 (a) Nonmydriatic photographs being defined as ungradeable (data from 178 eyes). (b) Mydriatic photograph being defined as ungradeable (data from 33 eyes). classified with poor image definition was predictive of a similar ungradeable mydriatic photograph $(P=0.001)$, however, the predictive value was poor at $34 \%$.

Binary logistic regression analysis was performed to determine which factors predicted the probability of obtaining an ungradeable nonmydriatic photograph (Table 3). Increasing age, posterior subcapsular cataract, and reduced near visual acuity achieved statistical significance as predictors of ungradeable mydriatic photographs. Diabetic control, duration of diabetes, reduced distance visual acuity, the presence of referrable retinopathy and other characteristics of any lenticular changes had no predictive value in the model. In patients with ungradeable nonmydriatic images, the numbers becoming gradeable after mydriasis were $71 \%(32 / 45)$ in those with posterior subcapsular cataract, $82 \%(139 / 170)$ in those over 60 years of age, and 79\% (103/131) in those with a visual acuity of $6 / 9$ or worse.

Features that predicted an ungradeable mydriatic photograph following an ungradeable nonmydriatic photograph include nuclear colour and poor definition of nonmydriatic image (Table 3).

\section{Discussion}

The assessment scheme we used allowed image definition, field, and visualisation of the fovea to be considered as independent variables. Other assessment systems in published reports have combined field and

Table 2 Characteristics of patients with incomplete data regarding photographic image quality (as subgroup of patients with unreadable undilated photo)

\begin{tabular}{|c|c|c|c|}
\hline & $\begin{array}{l}\text { Complete data } \\
\quad(\mathrm{n}=178)\end{array}$ & $\begin{array}{l}\text { Incomplete data } \\
\quad(\mathrm{n}=32)\end{array}$ & $\begin{array}{c}\text { P-value (Mann-Whitney } \\
\text { U-test* } ; \chi^{2} \text {-test }{ }^{\$} \text { ) }\end{array}$ \\
\hline Age $\left(\right.$ median $\left.\pm q_{\mathrm{L}}-q_{\mathrm{U}}\right)$ & $68(62-76)$ & $70(57-76)$ & $P=0.843^{*}$ \\
\hline Years since diagnosis (median $\pm q_{\mathrm{L}}-q_{\mathrm{U}}$ ) & $7(3-10)$ & $9(3-16)$ & $P=0.208^{*}$ \\
\hline Near visual acuity (median $\left.\pm q_{\mathrm{L}}-q_{\mathrm{U}}\right)$ & N5 (N5-N6) & N5 (N5-N8) & $P=0.626^{*}$ \\
\hline Lens nuclear colour ${ }^{13}\left(\right.$ median $\left.\pm q_{\mathrm{L}}-q_{\mathrm{U}}\right)$ & $2.0(1.5-2.8)$ & $2.3(0.5-3.0)$ & $P=0.544^{*}$ \\
\hline Posterior subcapsular cataract ${ }^{13}$ (median $\pm q_{\mathrm{L}}-q_{\mathrm{U}}$ ) & $0.2(0.2-0.65)$ & $0.2(0.2-2.4)$ & $P=0.279^{*}$ \\
\hline Presence of diabetic retinopathy & $36.5 \%$ & $53.1 \%$ & $P=0.071^{\$}$ \\
\hline Insulin dependant & $24.2 \%$ & $53.1 \%$ & $P>0.01^{\$}$ \\
\hline
\end{tabular}

Table 3 Factors predicting ungradeable status of nonmydriatic fundus Photograph, and mydriatic photograph following ungradeable nonmydriatic photograph (only statistically significant factors are tabulated)

\begin{tabular}{lccc}
\hline Nonmydriatic photography & Significance (P) & Odds ratio & 95\% confidence limits \\
\hline Age & $<0.001$ & 1.051 & $1.035-1.067$ \\
Posterior subcapsular cataract & 0.004 & 1.290 & $1.087-1.532$ \\
Near visual acuity & 0.006 & 1.141 & $1.039-1.253$ \\
& & & \\
Mydriatic photography & 0.006 & 1.944 & $1.205-3.137$ \\
$\quad$ Lens nuclear colour & 0.001 & 5.595 & $1.936-16.194$ \\
$\quad$ Ungradeable photograph due to poor definition & & & \\
\hline
\end{tabular}


definition together ${ }^{14,15}$ or image definition alone ${ }^{16}$ and usually do not address whether the fovea can be visualised as an independent criterion. We demonstrate the most common cause of an ungradeable image was the fovea not being adequately visualised, whether mydriasis was used or not. Poor image definition and less than $3 / 4$ of the field being visible were much less frequent causes of ungradeable images. Poor image definition on nonmydriatic photography was statistically associated with a similar outcome after mydriasis, but the predictive value was poor at $34 \%$, limiting its usefulness in the clinical setting.

The best predictors of ungradeable status of a nonmydriatic fundus photograph were patient age, the presence of posterior cataract, and poor near vision. For these patient groups, mydriasis made the images gradeable in $71-82 \%$ of patients. Unfortunately, we did not have the equipment ${ }^{17}$ to measure pupil size.

Age is not an independent risk factor. With increasing age there is an increase in lenticular changes, reduction in pupil size ${ }^{18}$ and increase in duration of diabetes.

Posterior subcapsular cataract on theoretical calculations is predicted to have the greatest impact on reducing nonmydriatic visual acuity due to the position at one of the nodal points of the eye. ${ }^{19}$ A dilated pupil reduces the impact of the localised subcapsular opacification.

Following mydriasis, nuclear colour (a measure of cataract severity) of the lens best predicted an ungradeable image from mydriatic fundus photograph. Patients with known nuclear cataract and/or previous failure with mydriatic photography could be referred directly for slit lamp examination in screening programmes. Nuclear cataract causes diffuse change of the lens and the fundus view is less likely to be improved with mydriasis.

Scanlon $e t a l^{20}$ recently published the results of a larger community-based study reporting on the influence of age, duration of diabetes, cataract, and pupil size on the image quality of digital photographs. While there were differences in the methodology and in the parameters assessed, results were similar. Scanlon also found increase in age and the presence of cataract decreasing the gradable rate of photographs. Cataract was measured on the basis of photographs while in our study the LOCS 111 scale $^{13}$ allowed the location and severity of the cataract to be compared. Scanlon measured pupil diameter on anterior segment photographs, cleverly using the same illumination that would be used for the screening photograph. By standarising magnification he was able to report on pupil diameter. A significant trend of decreasing pupil size associated with increasing rate of ungradable photograph was identified. No comment was made on the minimum pupil size required to obtain a gradable photograph, however, we believe this value would be variable dependant on other factors such as lenticular changes.

The use of mydriasis may impair the ability to drive and delay return to employment. ${ }^{10}$ In a study of 28 patients, all of whom had fulfilled the UK visual requirements for driving before dilating drops, $22 \%$ failed to read a standard number plate at $20 \mathrm{~m}$ indoors post dilation and $14 \%$ of patients felt unsafe to drive. ${ }^{11}$ A case report of a patient being involved in a road traffic accident following mydriatic retinal screening has highlighted some of the legal implications of driving following mydriasis. ${ }^{12}$ These are important considerations if using routine mydriasis.

Further work should be carried out to see whether mydriasis should be targeted towards patients over 60 years, with posterior subcapsular cataract and poor near vision. Young patients are more likely to be working and to appreciate the benefits of not undergoing pupillary dilatation.

\section{Acknowledgements}

We are grateful to the Ross Foundation and Tenovus Scotland in financially supporting this work.

\section{References}

1 Evans J, Rooney C, Ashwood F, Battani N, Wormald R. Blindness and partial sight in England and Wales. Health Trends 1996; 38: 5-12.

2 Moss SE, Klein R, Klein BE. The 14 year incidence of visual loss in a diabetic population. Ophthalmology 1998; 105: 998-1103.

3 James J, Turner D, Broadbent DM, Vora J, Harding S. Cost effectiveness analysis of screening for sight threatening eye disease. BMJ 2000; 320: 1627-1631.

4 Javitt JC, Aiello LP, Chiang Y, Ferris FL, Canner JK, Greenfield S. Preventive eye care in people with diabetes is cost-saving to the federal government. Diabetes Care 1994; 17: 909-917.

5 Gillow JT, Muir Gray JA. The National screening committee review of diabetic retinopathy screening. Eye 2001; 15: 1-2.

6 Williams GA, Scott IU, Haller JA, Maguire AM, Marcus D, McDonald R. Single-field fundus photography for diabetic retinopathy screening. A report by the American Academy of Ophthalmology. Ophthalmology 2004; 111: 1055-1062.

7 Facey K, Cummins E, Macpherson K, Morris A, Reay C, Slattery J. Organisation of Services for Diabetic Retinopathy Screening. Health Technology Assessment Report 1. Health Technology Board for Scotland: Glasgow, 2002. www.htbs.org.uk.

8 Scanlon PH, Malhotra R, Greenwood RH, Aldington SJ, Foy $\mathrm{C}$, Flatman $\mathrm{M}$ et al. Comparison of two reference standards in validating two field mydriatic digital photography as a method of screening for diabetic retinopathy. $\mathrm{Br} \mathrm{J}$ Ophthalmol 2003; 87: 1258-1263.

9 Murgatroyd H, Ellingford A, Cox A, Binnie M, Ellis JD, MacEwen CJ et al. Effect of mydriasis and different field 
strategies on digital image screening of diabetic eye disease. Br J Ophthalmol 2004; 88: 920-924.

10 Potamitis T, Slade S, Fitt A, McLaughlin J, Mallen E, Auld RJ et al. The effect of pupil dilation with tropicamide on vision and driving simulator performance. Eye 2000; 14: 302-306.

11 Goel S, Maharajan P, Chua C, Dong B, Butcher M, Bagga P. Driving ability after pupillary dilatation. Eye 2003; 17: 735-738.

12 Razvi S, Myers L, Patton K, McCulloch AJ. Screening for diabetic retinopathy: a cause for concern in people who drive. Diabet Med 2003; 20: 812-815.

13 Chylack LT, Wolfe JK, Singer DM, Leske MC, Bullimore MA, Bailey IL et al. The lens opacities classification system III. Arch Ophthalmol 1993; 111: 831-836.

14 Aldington SJ, Kohner EM, Meuer S, Klein R, Sjolie AK. Methodology for retinal photography and assessment of diabetic retinopathy: the EURODIAB IDDM complications study. Diabetologia 1995; 38: 437-444.

15 Olson JA, Strachan FM, Hipwell JH, Goatman KA, McHardy KC, Forrester JV et al. A comparative evaluation of digital imaging, retinal photography and optometrist examination in screening for diabetic retinopathy. Diabet Med 2003; 20: 528-534.

16 Williams R, Nussey S, Humphry R, Thompson G. Assessment of non-mydriatic fundus photography in detection of diabetic retinopathy. Br Med J 1986; 293: 1140-1142.

17 Schnitzler E, Baumeister M, Kohnen T. Scotopic measurement of normal pupils: Colvard versus Video Vision Analyser infrared pupillometer. J Cataract Refract Surg 2000; 26: 859-866.

18 Bitsios P, Prettyman R, Szabadi E. Changes in autonomic function with age: a study of pupillary kinetics in healthy young and old people. Age Ageing 1996; 25: 432-438.

19 Elkington AR, Frank HJ. Clinical Optics, 2nd ed., Chapter 9. Blackwell Science: Oxford, ISBN 0-632-03139-5, 1991.

20 Scanlon P, Foy C, Malhotra R, Aldington S. The influence of age, duration of diabetes, cataract, and pupil size on image quality in digital photographic retinal screening. Diabetes Care 2005; 28: 2448-2453. 